\title{
Simulating the Earth's Induction Effects on Substorm Data Recorded at Mid-Latitude Stations: The Three-Dimensional Problem
}

\author{
Marianne MARESChaL* and Jerry L. KisAbeth** \\ *Department of Physics, University of Toronto, Toronto, Canada \\ **Geophysics Department, Texas A\& M University, \\ College Station, Texas, U.S.A.
}

(Received September 16, 1976; Revised April 21, 1977)

\begin{abstract}
A major problem encountered when modeling external current systems from ground-based magnetic observations is the evaluation of the earth's induction effects. Though it is quite difficult to represent accurately the effects of induced currents flowing inside the earth, they can be approximated relatively easily under certain circumstances (i.e. when global rather than local electrical properties of the earth are concerned). For this purpose, two general classes of earth models can be used, i.e. (1) the models which reduce the earth to a superconductor (either at the surface or at some depth) and (2) the models in which the earth is represented by a body of finite conductivity (either uniform or layered). The performances of these two types of earth models are tested with respect to substorm modeling from mid-latitude data (i.e. when neither the curvature of the earth nor the three-dimensional nature of the substorm current system can be neglected). The conclusions are almost identical to those obtained previously with high latitude data (i.e. when the induction problem can be reduced to the study of a flat electrojet flowing over a flat earth). They indicate that for most problems of substorm modeling from ground-based magnetic observations, it is sufficient to treat the earth as a superconductor ("perfect" conductor).
\end{abstract}

\section{Introduction}

During the last decade, it has become more and more apparent to substorm researchers that modeling an external current system to fit magnetic observations recorded at the earth's surface requires an adequate evaluation of the earth's induction effects. Such an evaluation is quite difficult because it depends on the knowledge of both the external current system distribution and the earth's conductivity structure. However, it can be approximated rather easily when the induction problem only involves the global properties of the earth, i.e. when its lateral and surface inhomogeneities are neglected. 
For that purpose, two general classes of earth models can be used; (1) the models which reduce the earth to a superconductor (either at the surface or at some depth) and (2) the models in which the earth is represented by a body of finite conductivity (either uniform or "horizontally" layered).

THE TWO-DIMENSIONAL PROBLEM: A FLAT ELECTROJET OVER A FLAT EARTH
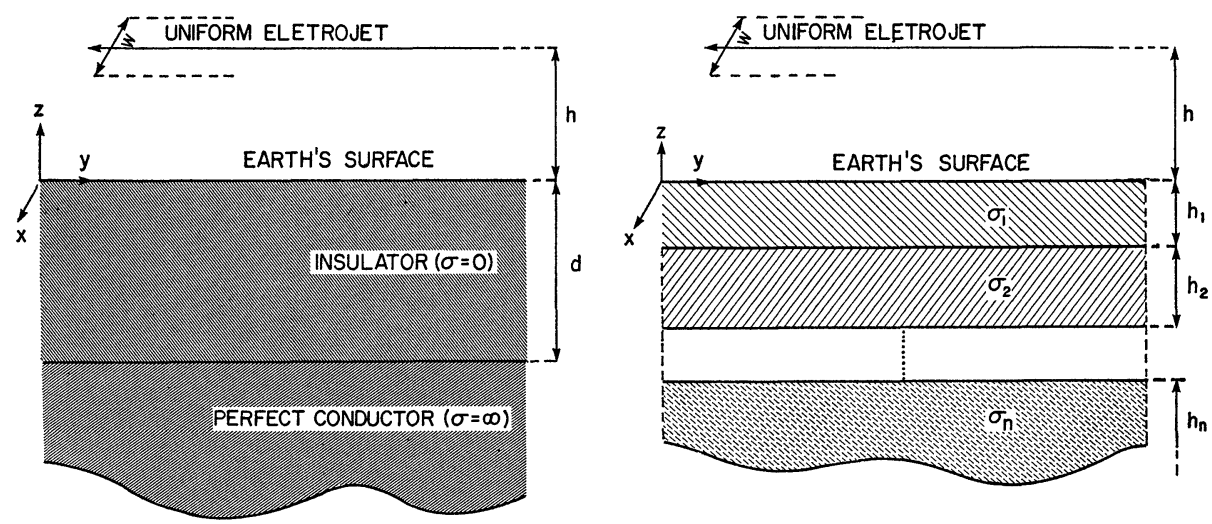

Fig. 1. The two-dimensional problem studied by Mareschal (1976). The magnetic signatures of an electrojet at the surface of a flat earth (either modeled as a perfect conductor (left) or as a finite conductor (right)) are evaluated for comparison.

THE THREE DIMENSIONAL PROBLEM: A T.D. CURRENT SYSTEM OVER A SPHERICAL EARTH
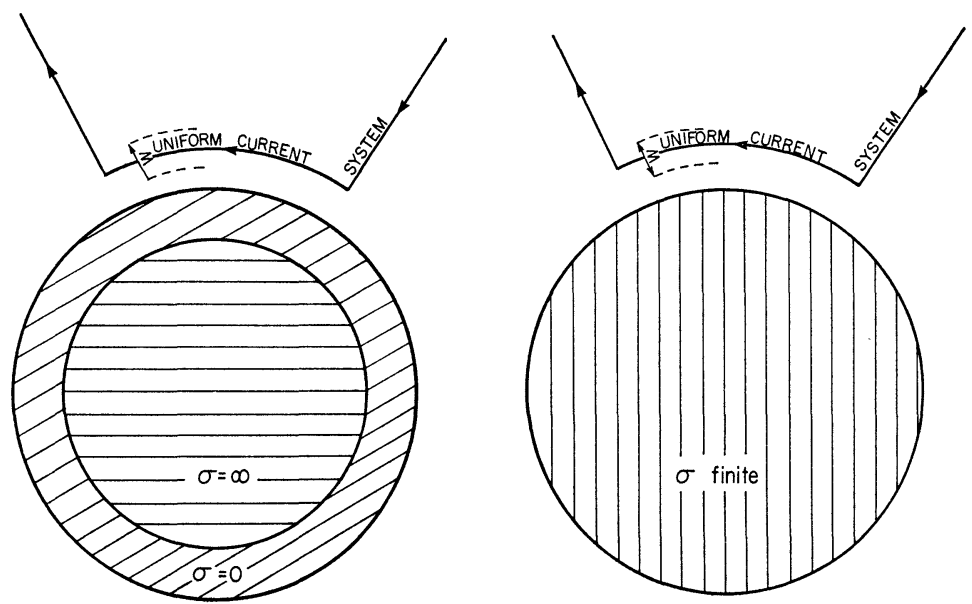

Fig. 2. The three-dimensional problem. The magnetic signatures of a three-dimensional current system at the surface of a spherical earth (either modeled as a perfect conductor (left) or as a finite conductor (right)) are evaluated for comparison. 
The preformances of these two types of earth models have recently been tested with respect to substorm modeling from high latitude magnetic observations (MARESCHAL, 1976). Mareschal concluded that, in general, it was sufficient to treat the earth as a perfect conductor to simulate its response to a magnetic substorm (the advantage of such a representation being, of course, that it leads to very simple field equations). However, she restricted her discussion to the two-dimensional problem, i.e. to the study of the surface signature of a flat electrojet inducing horizontal currents in a flat earth (see Fig. 1).

But if the substorm data selected for the modeling are recorded at midlatitude stations, neither the curvature of the earth nor the three-dimensional nature of the substorm current system itself can be neglected in the resolution of the induction problem (e.g. see Cloutier, 1971; Armstrong and ZMUda, 1973; Chappell, 1974; Anderson and VondraK, 1975; Potemra and Iijima, 1976 for observations of the substorm current system). It is thus mandatory to verify that Mareschal's conclusions remain true in that context, i.e. to show that, indeed, the earth induction effects on mid-latitude substorm data are satisfactorily simulated by the response of a superconducting sphere to a three-dimensional external current system (see Fig. 2). This is the purpose of this paper.

\section{Mathematical Formulation of the Induction Problem}

Only when the geometrical parameters characteristic of an induction problem are very much simplified (i.e. simple current systems flowing over a flat, horizontally-layered earth) can an exact solution to the problem be obtained relatively easily. When the curvature of the earth cannot be neglected, the solution of the induction problem is usually expressed in terms of spherical harmonics because it is assumed that the source field is derivable from a known potential satisfying Laplace equation (e.g. LAHIRI and PRICE, 1939). Unfortunately, in most problems of substorm modeling, that potential is not a priori known. The source field elements are obtained numerically from a geometrical model of current system and their expansion in spherical harmonics is not performed.

This consideration led Ashour (1971) to present a method for determining the field induced in a spherical earth from the knowledge of the numerical expression of the source field alone. For that purpose, he chose to simulate the earth's electrical properties by introducing a thin shell of high conductivity at some depth below the earth's surface. Although the source field's expression in spherical harmonics was not explicitly needed, the method was only applicable to fields characterized by harmonics of low degree.

The same principle is used in Appendices 1 and 2. We, too, only consider 
external fields whose spherical harmonic representation would involve harmonics of low degree. But we extend Ashour's approach to more realistic earth models, i.e. (1) to models in which the earth is represented by a sphere of uniform and finite conductivity (Appendix 1) and (2) to two-layered earth models consisting of highly conducting central core surrounded by a less conducting shell (Appendix 2). Because of the earth models selected, our approach necessitates a more general treatment than Ashour's and is based on the mathematical theory of induction by varying magnetic fields in regions bounded by concentric spheres (e.g. Chapman and Bartels, 1940). Ashour's result (for a superconductor) is obtained as a particular case of the general solution.

That solution is given as an infinite series whose terms involve increasing powers of $1 / k a$ (where $k$ depends on both the earth model and the current system model while $a$ is the earth radius). For instance

$$
\begin{aligned}
B_{r}^{i}(r=a, \theta, \phi)= & -B_{r}^{e}(r=a, \theta, \phi)+\frac{1}{k a}\left[\frac{2}{r^{1 / 2}} \frac{\mathrm{d}}{\mathrm{d} r} r^{3 / 2} B_{r}^{e}(r, \theta, \phi)\right]_{r=a} \\
& +\frac{1}{(k a)^{2}}\left[\frac{\mathrm{d}}{\mathrm{d} r} r B_{r}^{e}(r, \theta, \phi)-2 \frac{\mathrm{d}^{2}}{\mathrm{~d} r^{2}} r^{2} B_{r}^{e}(r, \theta, \phi)\right]_{r=a} \\
& +\cdots \\
& \text { (Appendix 1) }
\end{aligned}
$$

(the superscripts $i$ and $e$ stand respectively for induced and external (or source) and $\boldsymbol{B}$ is the magnetic induction).

Therefore, if the source field components are known numerically along the radius of the earth to its surface, the corresponding induced field components can be determined at the earth's surface. The method usually does not increase the total computer time necessary to evaluate the external field alone by more than $10 \%$. Note that the first term, which does not depend on $k$, is the exact solution to the three-dimensional problem of induction in a superconducting earth. When only the central core of the earth $(r=b)$ is assumed to be superconducting, that term becomes

$$
B_{r}^{i}(r=a, \theta, \phi)=-\xi^{3} B_{r}^{e}\left(r=\xi^{2} a, \theta, \phi\right) \quad(\text { Ashour, 1971) }
$$

where $\xi=b / a$. Similar transformations would affect $B_{\theta}^{i}$ and $B_{\phi}^{i}$ obtained in Appendix 1 (Eqs. (A.20) and (A.21), zeroth order of approximation).

\section{The Model Current System}

An explicit application of the results presented in section 2 requires, of course, the choice of a model current system allowing the determination of $\boldsymbol{B}^{\diamond}$ (the source field). The model selected in this study consists of Birkeland currents flowing down the field lines to the ionosphere, westward along the electro- 
jet and back up again in the magnetosphere (see Fig. 3). This model has been used as an equivalent current system for isolated substorms by numerous authors and, in particular, was shown to generate perturbations which were in good agreement with the magnetic disturbances observed during a polar magnetic substorm (e.g. BonNevier et al., 1970; KISABETH and Rostoker, 1971, 1973, 1974, 1977; Rostoker and Kisabeth, 1973).

The mathematical expressions of the field components $\left(B_{r}^{e}, B_{\theta}^{e}, B_{\phi}^{e}\right)$ have been

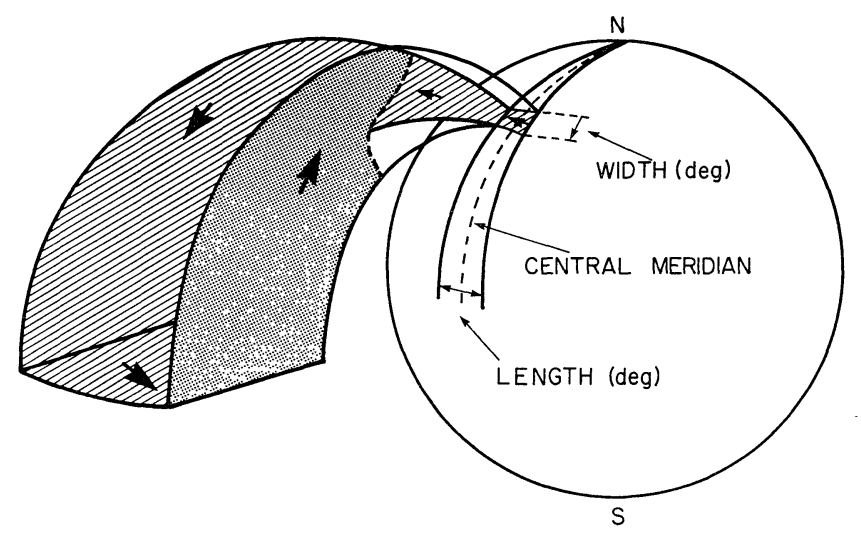

Fig. 3. Model of a three-dimensional E-W current system with finite longitudinal and latitudinal extents (after KisABETH, 1972).

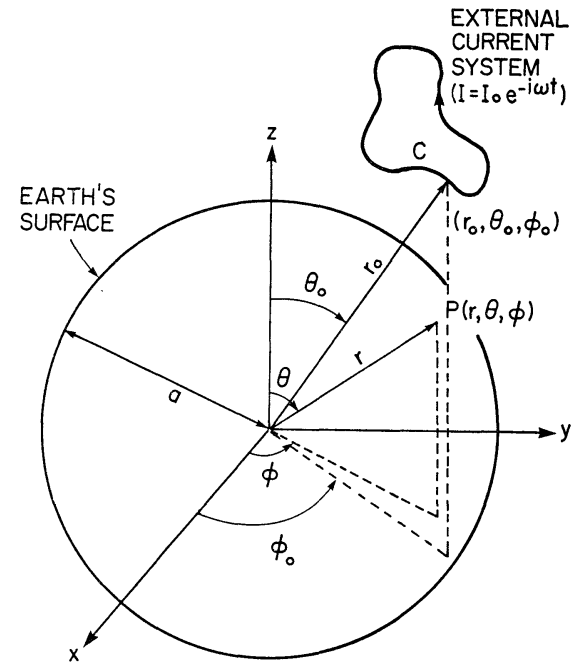

Fig. 4. Diagram showing the geometry and the system of coordinates used in section 4 for the magnetic field calculations. 
presented by Kisabeth (1972) (see also Kisabeth and Rostoker, 1977) in the form of a compact matrix equation. Kisabeth's simple formulation was obtained from the application of the generalized Biot-Savart law and is reproduced below.

Assuming that $B_{r}^{e}, B_{\theta}^{e}$ and $B_{\phi}^{e}$ represent the components of the magnetic induction in the observation coordinate system $(r, \theta, \phi)$ whereas $\mathrm{d} S_{1}, \mathrm{~d} S_{2}$ and $\mathrm{d} S_{3}$ are the components of the differential current path $C$ in the source coordinate system $\left(r_{0}, \theta_{0}, \phi_{0}\right)$ (see Fig. 4$)$, Kisabeth's solution can be written as

$$
\left[\begin{array}{c}
B_{r}^{e} \\
B_{\theta}^{e} \\
B_{\phi}^{e}
\end{array}\right]=\frac{\mu_{0} I}{4 \pi} \int_{C}\left[\begin{array}{ccc}
0 & f A_{13} & -f A_{12} \\
g A_{31} & \left(f A_{23}+g A_{32}\right) & \left(g A_{33}-f A_{22}\right) \\
g A_{12} & \left(f A_{33}-g A_{22}\right) & \left(-g A_{23}-f A_{32}\right)
\end{array}\right]\left[\begin{array}{c}
\mathrm{d} S_{1} \\
\mathrm{~d} S_{2} \\
\mathrm{~d} S_{3}
\end{array}\right] .
$$

In this expression, the matrix $A$ relates the observation and source coordinate systems by

$$
X=A X_{0}
$$

while the terms $f$ and $g$ are defined as

and

$$
f=r / R^{3}
$$

with

$$
g=r_{0} / R^{3}
$$

$$
R=\left(r^{2}+r_{0}^{2}-2 r r_{0} A_{11}\right)^{1 / 2} .
$$

Note that KISABETH (1972) already generalized this matrix equation to take into account the earth induction effects (following Ashour's approach, i.e. the earth is a superconductor). Most of his papers referred to above use the generalized formulation.

\section{Range of Application of Our Solution to the Induction Problem}

Since $\boldsymbol{B}^{e}$ (the source field) is known from Eq. (3), the exact solution to the problem of induction in a superconductor can be determined through Eq. (2) and its extensions derived in Appendix 1.

However, our mathematical solution to the problem of induction in a finite conductor requires the use of an infinite series which, in practice, has to be truncated after a certain number of terms (see Eq. (1) and its extensions in Appendix 1). The advantage of such a representation should be obvious to substorm researchers since it only requires a small amount of computer time to model the earth induction effects and therefore allows the sophistication of the current system itself. Nonetheless, its limitations must not be forgotten. The conditions under which the series truncation is allowed have thus to be defined without ambiguity before the approximation is used. This is done in this section. 
Table 1. Determination of Real $(k r)$ at $r=6,371 \mathrm{~km}$ (earth's surface).

\begin{tabular}{lrr}
\hline & \multicolumn{2}{c}{$\sigma$ (e.m.u.) } \\
\cline { 2 - 3 } & $10^{-14}$ & $10^{-13}$ \\
\hline $2 \pi / 60$ & 51.7 & 163.4 \\
$2 \pi / 900$ & 13.3 & 42.2 \\
$2 \pi / 3,600$ & 6.7 & 21.1 \\
\hline
\end{tabular}

It must be clear, from the reading of both appendices that our representation of $\boldsymbol{B}^{i}$ (the induced field) is based on the assumption that the real part of some realistic " $k r$ " is large when compared to $n$, the degree of the harmonics which would be necessary to represent the source field at the earth's surface.

Since $k^{2}$ is defind as $k^{2}=-4 \pi \sigma \mu i \omega$ (Appendix 1) and since $r$ is real ( $r$ is the radius of a sphere), Real ( $k r)$ can be expressed as

$$
\operatorname{Real}(k r)=\sqrt{2 \pi \mu \sigma \omega} r .
$$

The magnitude of such a $\operatorname{Real}(k r)$ is given in Table 1 for some realistic values of $\sigma$ (the earth conductivity) and $\omega$ (the substorm radian frequency). Real ( $k r)$ is evaluated at the surface of a uniform earth, $\sigma$ being selected as the average conductivity of the upper mantle and $r=6.371 \mathrm{~km}$ (see Fig. Al).

Note first that since $n=35$ does not even come close to reproducing substorm fields in the auroral regions, our method of evaluating $\boldsymbol{B}^{i}$ is only valid for mid-latitude and low latitude stations.

Note second that for the high frequency components of the field, the zeroth order approximation is certainly quite satisfactory. Indeed, whatever the value $n$ assumes for mid-latitude substorm fields, $(2 n+1) /$ Real $(k r)$ is probably negligible when compared to 1 (see Eq (A.17)).

Note then that even the low frequency components of the field should not require a higher order of approximation than the second order terms to describe $\boldsymbol{B}^{i}$. If a value of 6.7 for Real $(k r)(T=1 \mathrm{hr})$ appears to be too small when compared to $n$ to allow that order of approximation, further terms can be calculated. However, it shoud be remembered that (1) an average conductivity of $10^{-14}$ e.m.u. $\left(10^{-3} \mathrm{~s} / \mathrm{m}\right)$ is probably too small to be representative of the earth mantle (e.g. see BANKs (1972) for a recent review of global conductivity models) and could be increased by an order of magnitude without altering the physical significance of the result (in which case Real $(k r)=21.1)$ and that $(2)$ our formalism is supposed to be applied to mid-latitude data where the field is much smoother than in the auroral regions ( $n$ effective should be relatively small).

Note also that since the actual earth can seldom be realistically represented 
Table 2. Determination of Real $(k r)$ at $r=5,871 \mathrm{~km}$.

\begin{tabular}{lrr}
\hline & \multicolumn{2}{c}{$\sigma($ e.m.u. } \\
\cline { 2 - 3 } & $10^{-11}$ & $10^{-10}$ \\
\hline $2 \pi / 900$ & 388.8 & $1,229.6$ \\
$2 \pi / 3,600$ & 194.4 & 614.8 \\
\hline
\end{tabular}

Table 3. Skin depth (in $\mathrm{km}$ ) for various earth conductivities and source frequencies.

\begin{tabular}{lcc}
\hline & \multicolumn{2}{c}{$\sigma$ (e.m.u.) } \\
\cline { 2 - 3 } & $10^{-14}$ & $10^{-13}$ \\
\hline $2 \pi / 900$ & 477 & 151 \\
$2 \pi / 3,600$ & 955 & 302 \\
\hline
\end{tabular}

by a sphere of uniform conductivity, a two-layered model has been introduced in Appendix 2 (see Fig. A2). To support the approximations introduced in that appendix, Real $(k r)$ is evaluated at the surface of a highly conducting core in Table 2. In this table, $r=5,871 \mathrm{~km}$, i.e. the model is supposed to be representative of the jump in conductivity present roughly $500 \mathrm{~km}$ below the earth's surface.

Obviously, Real $(k r)$ is always large enough in the central core to legitimize the zeroth order approximation which was used in that region (see Appendix 2). However, it should be remembered that the use of a two-layered earth model is only legitimate if a substantial fraction of the current induced inside the earth can reach the depth of the interface; the lower their frequency, the deeper the currents will flow (see Table 3). A two-layered earth model should not, therefore, be of any interest for modeling the earth's magnetic response to the high frequency components of the substorm field unless some very conducting anomalies close to the earth's surface have to be considered. Unfortunately, the lower the source frequency, the smaller Real $(k r)$ (for a specific choice of $\sigma$ ) and therefore the higher the degree of approximation required to simulate the field induced in the shell surrounding the central core. It should not be forgotten that the solution presented in Appendix 2 only makes use of the zeroth and first order terms in that region.

Note finally that whatever the earth model considered, Real $(k r)$ is always large enough to allow the truncation of $F_{n}\left(k^{2} r^{2}\right)$ (Eq. (A.8)) as was done in Appendix 1. Even if Real $(k r)=6.7$, the proportionate error between the expression of $F_{n}$ approximate and $F_{n}$ actual is

$$
e^{-2 k r} \rho_{n}(-k r) / \rho_{n}(k r) \text {. }
$$

Since Real $\left(e^{-2 k r}\right)=e^{-13.4}$, the error could only be large if $\rho_{n}(-k r) / \rho_{n}(k r)$ was 
extremely large which is in contradition with our assumption that $n$ is small when compared to Real ( $k r)$ (see the series expansion of $\rho_{n}$, i.e. Eq. (A.12)).

Of course, this purely mathematical discussion of the relative magnitudes of Real $(k r)$ and $n$ might appear quite immaterial since $n$ is unknown in our representation. It can only be guessed if we do not want to go through the process of expanding the field in spherical harmonics. Fortunately, this problem is not crucial since, because of the interrelationship between the degree of the harmonics necessary to represent a field and its smoothness, the condition on $n / k r$ can be replaced by a condition on the consecutive derivatives of the source field (see Appendix 1 or Eq. (1)). This supports our suggestion that our solution should be appropriate to mid-latitude stations where the substorm field varies relatively smoothly. In that ragion, $n$ effective is normally small and our representation should describe $\boldsymbol{B}^{i}$ adequately for source frequencies up to $2 \pi / 3,600 \mathrm{sec}^{-1}$.

To substantiate this point, the two first terms (zeroth and first orders) of the induced field components have been calculated at various mid-latitude stations using Eq. (1) and extensions, The results are presented in Table 4. To be consistent with our previous analysis in terms of a series development in Real $(\mathrm{kr})$, we only give the real part of $\boldsymbol{B}^{i}(1 \mathrm{st})$ in this table $\left(\boldsymbol{B}^{i}(1 \mathrm{st})\right.$ is a complex number). $\boldsymbol{B}^{i}(2 \mathrm{nd})$ being purely imaginary, a further convergence test would require the calculation of Real $\left(\boldsymbol{B}^{i}(3 \mathrm{rd})\right)$. This is not done here. However, the amplitude of $\boldsymbol{B}^{i}(2 \mathrm{nd})$ has been calculated and was found to be small enough when compared to the amplitude of $\boldsymbol{B}^{i}(1 \mathrm{st})$ to indicate a rapid convergence of the series representing the induced field at mid-latitude stations.

In this example, the earth is simulated by a sphere of uniform conductivity $\left(\sigma=10^{-13}\right.$ e.m.u. or $\left.10^{-2} \mathrm{~s} / \mathrm{m}\right)$ and the three-dimensional current system is defined by the following electrojet: height above the earth's surface $(115 \mathrm{~km})$, width $\left(4.3^{\circ}\right.$ of latitude), length $\left(59.5^{\circ}\right.$ of longitude $)$, moment $\left(68.3^{\circ}\right.$ of latitude) and maximum current intensity $\left(0.59 \times 10^{6} \mathrm{~A}\right)$. The electrojet's central meridian is selected as reference meridian $\left(0^{\circ}\right.$ of longitude) and the current intensity varies sinusoidally with a period of $1 \mathrm{hr}$ (see Fig. 3 for a definition of the current system).

Table 4. Evaluation of Real $\left(\boldsymbol{B}^{i}\right)$ at various mid-latitude stations $(\omega=$ $\left.2 \pi / 3,600 \mathrm{sec}^{-1}\right)$.*

\begin{tabular}{lrrrrrrrr}
\hline \multirow{2}{*}{$\begin{array}{c}\text { Lat., long. } \\
\text { of the station }\end{array}$} & \multicolumn{3}{c}{ Zeroth order } & & \multicolumn{3}{c}{ 1st order } \\
\cline { 2 - 6 } \cline { 6 - 8 } & $B_{\theta}^{i}$ & $B_{\dot{\phi}}^{i}$ & $B_{r}^{i}$ & & $B_{\theta}^{i}$ & $B_{\dot{\phi}}^{i}$ & $B_{r}^{i}$ \\
\hline $35^{\circ},-75^{\circ}$ & 1.9 & 4.4 & 2.1 & & -0.6 & -0.4 & -0.2 \\
$41^{\circ},-55^{\circ}$ & 1.7 & 8.6 & -0.7 & & -0.5 & -1.0 & -0.3 \\
$47^{\circ},-35^{\circ}$ & -2.0 & 16.6 & -9.8 & & 10.3 & -0.9 & 0.03 \\
$53^{\circ},-15^{\circ}$ & -18.4 & 21.4 & -37.4 & & 149.7 & 8.7 & 2.4 \\
\hline
\end{tabular}

* The field is expressed in $\gamma$. 
Note first that our selection of $\omega\left(\omega=2 \pi / 3,600 \mathrm{sec}^{-1}\right)$ leads to relatively small values of $k r(\operatorname{Real}(k r)=21.1$, see Table 1$)$. Our test would obviously lead to more satisfactory conclusions if a higher source frequency had been used.

Note then that the horizontal component of the field is less stable than any other component. At the third station $\left(47^{\circ},-35^{\circ}\right)$, its first order term is already larger (in amplitude) than its zeroth order term. Other tests give the same indication (latitudinal and longitudinal effects have been investigated). Obviously, our representation of $\boldsymbol{B}^{i}$ in terms of a truncated series is not valid in the high latitude regions. However, this is not a restriction on the method itself since it has only been introduced for regions where the sphericity of the earth and the three-dimensional nature of the substorm current system could not be neglected. In the high latitude regions, the two-dimensional representation (flat electrojet over a flat earth) leads to a quite satisfactory solution.

In conclusion, it appears that our solution to the induction problem is appropriate to the purpose that we have in mind, i.e. to compare the mid-latitude signatures a three-dimensional current system at the surface of either a superconducting or a finitely conducting earth. This comparison is thus performed in the following section.

5. Comparison between the Possible Signatures of a Substorm Field at the Surface of either a Perfectly or a Finitely Conducting Earth

Computationally, simulating the earth by a superconductor has two enormous advantages for the substorm researcher. First, it generates extremely simple field equations which are entirely independent of the source frequency (see Eq. (2)). Second, as a consequence, the induced and source fields are in phase. Therefore, there is no time lag between both contributions. The instantaneous signature of the substorm (direct + induced contributions) can be evaluated at any moment without having to bother about causality relationships. Unfortunately, such a representation is not physically realistic.

Physically, simulating the earth by a finite conductor (either uniform or layered) is much more satisfactory. However, that representation requires a much larger amount of computer time and a much better knowledge of the source structure than the representation of the earth as a superconductor. Indeed, the field equations are frequency dependent (see Eq. (1)). Therefore, the total field has to be evaluated at each point of the stations array for every sample of frequency contained in the source. The source and induced contributions are not in phase and serious time lags can appear between them. The evaluation of the substorm's signature (total field) is a causal problem which usually requires the use of Fourier transforms. 
To support the suggestion of MARESCHAL (1976) that for most problems of substorm modeling from ground-based magnetic observations, the earth can be represented by a superconductor, three examples are presented below.

In the first example, the intensity of the current system already defined (see section 4 and Fig. 3 ) is

$$
I=I_{0} \cos \omega t
$$

where

$$
I_{0}=10^{6} \mathrm{~A} \quad \text { and } \quad \omega=2 \pi / 900 \mathrm{sec}^{-1} \quad\left(\sigma=10^{-3} \mathrm{~s} / \mathrm{m}\right) .
$$

Its pseudo signatures are evaluated at a mid-latitude station $\left(54^{\circ}\right.$ of latitude, $-60^{\circ}$ of longitude) situated either at the surface of a superconducting sphere or at the surface of a sphere of uniform finite conductivity (see Fig. 2). Obviously, these signatures should differ in amplitude and phase. However, it is very easy to adapt the dimension of the superconducting core in the first representation in such a way as to bring both amplitudes close to one another. The phase difference cannot be changed since it depends on physical parameters such as the earth conductivity $(\sigma)$ and the source frequency $(\omega)$.

In this first example, it is shown that the amplitudes of both signatures come close if $d$ (the depth of the superconducting core inside the earth) is chosen to be $100 \mathrm{~km}$ (see Fig. 5 where $Z=-B_{r}, H=-B_{\theta}$ and $D=B_{\phi}$ have been plotted). This effective depth $d$ would be different if the source frequency was varied (e.g. if $\omega=2 \pi / 3,600 \mathrm{sec}^{-1}$, the best fitting value of $d$ is $210 \mathrm{~km}$; if $\omega=2 \pi / 60 \mathrm{sec}^{-1}$, the best fitting value of $d$ is $35 \mathrm{~km}$ ). But the same Fig. 5 also shows that $t_{z}$ is relatively important $\left(t_{Z}\right.$ is the absolute value of the time lag between the vertical components of the fields measured at the surface of either a superconducting or a finitely conducting earth). However, $t_{H}$ and $t_{D}$ are negligible. This very same observation was made by MARESCHAL (1976) in her analysis of the twodimensional problem and is the major weakness of the representation of the earth as a superconductor. Note that the time lags $\left(t_{Z}, t_{H}\right.$ and $\left.t_{D}\right)$ can correspond to either a lead or lag of $\boldsymbol{B}$ (finite conductor) depending on the position of the observer as was already pointed out by Nopper and Hermance (1974).

Figure 6 deals with exactly the same problem. Only the station where the pseudo-data are recorded has been changed ( $35^{\circ}$ of latitud, $-20^{\circ}$ of longitude). It shows that our conclusions remain unchanged in a region where the signs of the field components are reversed. The best fitting $d$ is still $100 \mathrm{~km}$ for a source frequency $\omega=2 \pi / 900 \mathrm{sec}^{-1}$ and $t_{Z}$ is still difficult to neglect.

The final example concerns a more realistic current system, i.e. a system in which the current intensity builds up linearly with time and decreases similarly (triangular function, total duration $=1 \mathrm{hr}$ ). A Fourier decomposition of the source is then necessary to determine its frequency content and to allow the 


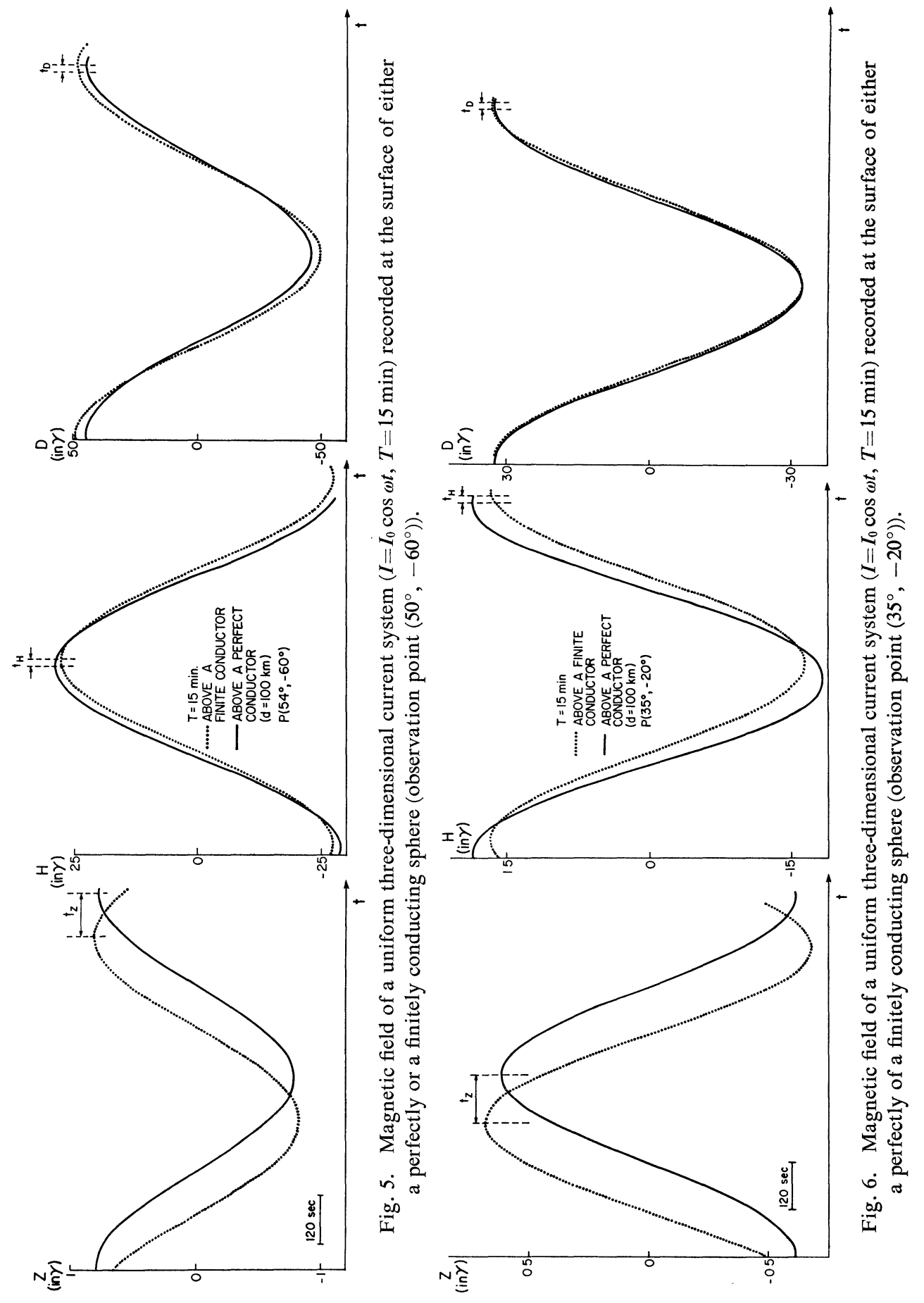


Simulating the Earth's Induction Effects

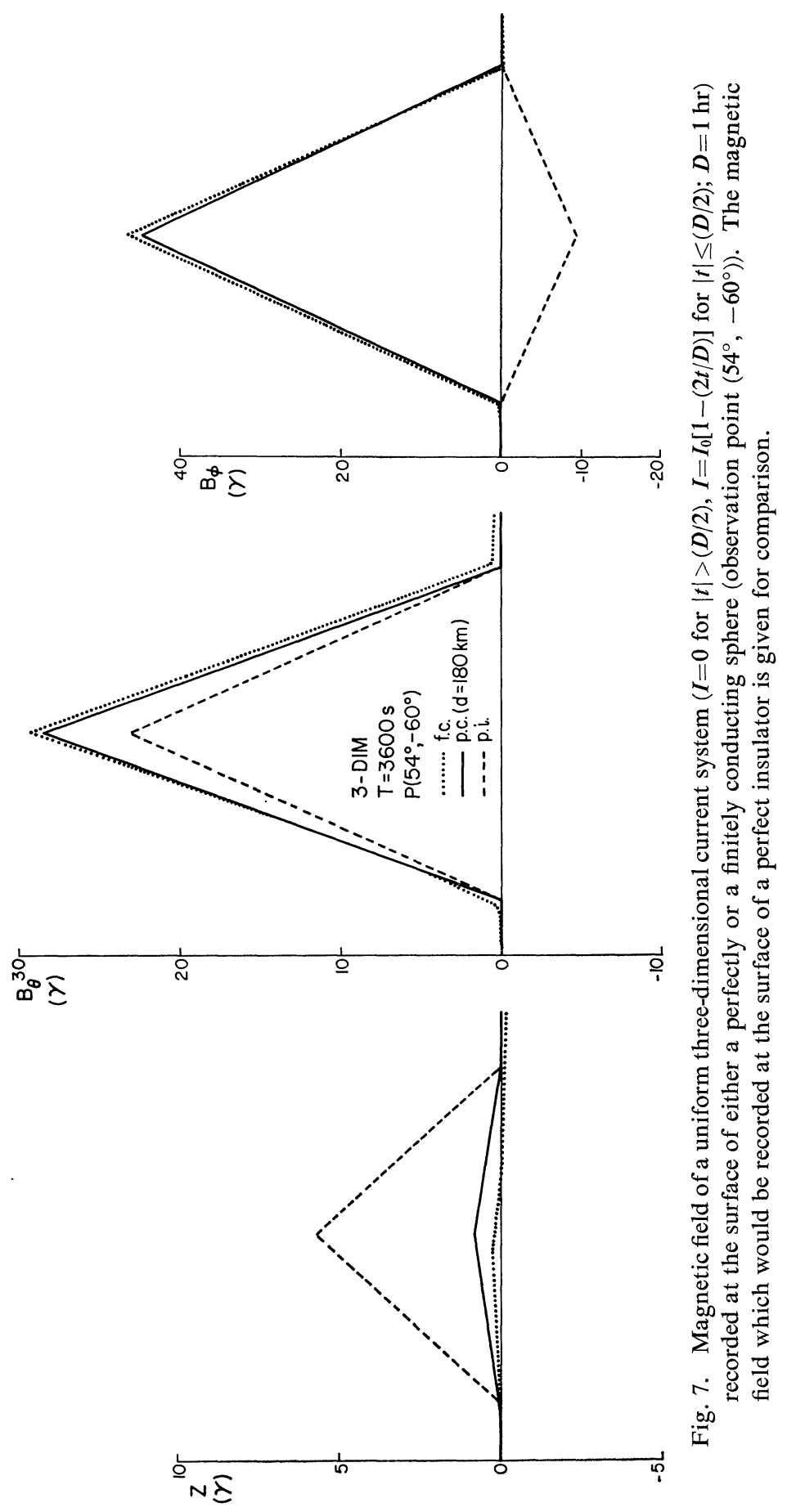


field evaluation above a finite conductor $\left(\sigma=5 \times 10^{-3} \mathrm{~s} / \mathrm{m}\right)$. The results are transformed back to the time domain and compared to the field which would be measured at the surface of a superconductor (see Fig. 7 where $Z\left(=-B_{r}\right.$ ), $B_{\theta}$ and $B_{\phi}$ are plotted for the station used in our first example $\left(54^{\circ}\right.$ of latitude, $-60^{\circ}$ of longitude)). The figure also shows (in dashed lines) the signature of the same current system as it would be recorded at the surface of a perfect insulator (i.e. the source field alone). Obviously, neglecting the earth's electrical properties leads to aberrant results. Clearly, too, the only significant difference between the responses of a superconducting or a finitely conducting earth still appear in the vertical component of the field.

\section{Conclusion}

The purpose of this article was to verify that MARESCHAL's conclusions (1976) remained true when passing from the two-dimensional induction problem to the three-dimensional problem. Indeed, the results that she obtained when studying the field induced by a flat electroject in a flat earth are almost identical to those presented in this paper where we consider the field induced in a spherical earth by a three-dimensional current system. They indicate that the earth can satisfactorily be modeled as a super conductor to simulate its response to a substorm field (except, perhaps, for the vertical component of that field).

The use of a more elaborate representation (the earth as a finite conductor) should probably only be necessary when the modeling of a specific station's response to various external current systems is desired. As far as the numerical inversion of substorm data recorded at the earth's surface is concerned (e.g. Czechowsky, 1971; Kisabeth and Mareschal, 1974; Horning et al., 1974; Oldenburg, 1976) a superconducting earth model should probably be quite appropriate. The best fitting depth for the superconductor below the earth's surface must be implicitely evaluated in function of the dominant source frequency. Note that this equivalent superconductor has usually to be placed deeper below the surface of a flat earth than below the surface of a spherical earth (same conductivity model) to generate the same field at the surface.

Appendix 1. Evaluation of the Field Induced by an External Current System of Known Geometry in a Sphere of Uniform Conductivity

Though the fundamentals of the method can be found in CHAPMAN and BARTEL's "Geomagnetism" (1940, p. 732-741), they are summarized in the first section of this appendix to improve the consistency of our development. The original work should be consulted for a more detailed presentation. 
A1. General expression of the magnetic induction $\boldsymbol{B}$ in regions bounded by spheres (after CHAPMAN and BARTELS, 1940)

Let us consider a conducting sphere (of uniform conductivity $\sigma$ and magnetic permeability $\mu$ ) surrounded by a non-conducting medium $\left(\sigma=0, \mu=\mu_{0}\right)$ (see Fig. A1).

In the non-conducting region, the magnetic induction $\boldsymbol{B}$ is derivable from a scalar magnetic potential $\Omega$ given by

$$
\Omega=\sum_{n, m} a\left(\rho^{n} E_{n}^{m}+\rho^{-n-1} I_{n}^{m}\right) S_{n}^{m}(\theta, \phi)
$$

where $a$ is a constant (length).

$S_{n}^{m}(\theta, \phi)$ is a spherical surface harmonic of degree $n$ and order $m$ and

$$
\rho=r / a
$$

(It is assumed that $E_{n}^{m}$ and $I_{n}^{m}$ are functions of time only and $\sum_{n, m}$ stands for $\left.\sum_{n=0}^{\infty} \sum_{m=0}^{+n}\right)$.

The components of $\boldsymbol{B}$ can therefore be expressed very simply. For instance

$$
B_{r}=-\mu_{0} \frac{\partial \Omega}{\partial r}=B_{r}^{e}+B_{r}^{i}
$$

where

$$
B_{r}^{e}=-\mu_{0} \sum_{n, m} n E_{n}^{m} \rho^{n-1} S_{n}^{m}(\theta, \phi)
$$

and

$$
B_{r}^{i}=+\mu_{0} \sum_{n, m}(n+1) I_{n}^{m} \rho^{-n-2} S_{n}^{m}(\theta, \phi) .
$$

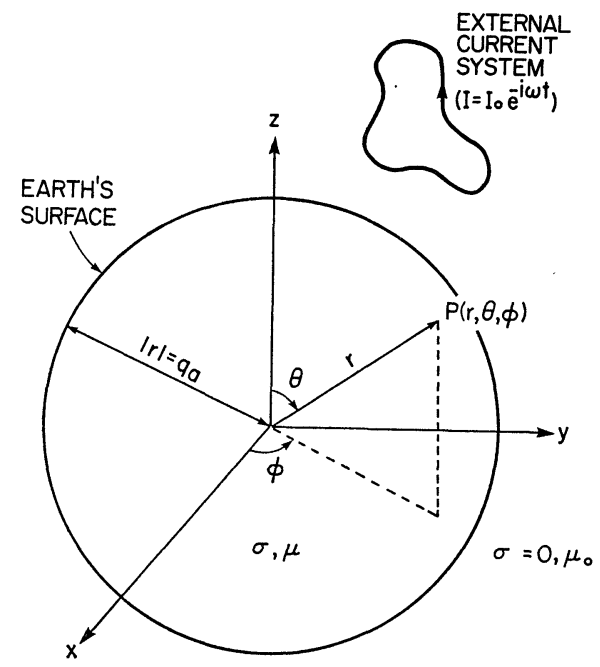

Fig. A1. Cross-section of a uniform earth with indication of the observation point. 
Similarly

where

$$
B_{\theta}=-\mu_{0} \frac{\partial \Omega}{r \partial \theta}=B_{\theta}^{e}+B_{\theta}^{i}
$$

and

$$
B_{\theta}^{e}=-\mu_{0} \sum_{n, m} E_{n}^{m} \rho^{n-1} \frac{\partial S_{n}^{m}(\theta, \phi)}{\partial \theta}
$$

$$
B_{\theta}^{i}=-\mu_{0} \sum_{n, m} I_{n}^{m} \rho^{-n-2} \frac{\partial S_{n}^{m}(\theta, \phi)}{\partial \theta}
$$

and finally

$$
B_{\phi}=-\mu_{0} \frac{\partial \Omega}{r \sin \theta \partial \phi}=B_{\phi}^{e}+B_{\phi}^{i}
$$

where

$$
B_{\dot{\phi}}^{e}=-\mu_{0} \sum_{n, m} E_{n}^{m} \rho^{n-1} \frac{\partial S_{n}^{m}(\theta, \phi)}{\sin \theta \partial \phi}
$$

and

$$
B_{\phi}^{i}=-\mu_{0} \sum_{n, m} I_{n}^{m} \rho^{-n-2} \frac{\partial S_{n}^{m}(\theta, \phi)}{\sin \theta \partial \phi} .
$$

On the other hand, inside the conducting region, the vector potential $\boldsymbol{A}$ satisfies

$$
\nabla^{2} A+4 \pi \sigma \mu i \omega A=0
$$

(the time dependence of the field is supposed to be of the form $e^{-i \omega t}$ ). Letting

$$
k^{2}=-4 \pi \sigma \mu i \omega
$$

the components of the magnetic induction $B$ which satisfy

$$
\boldsymbol{B}=\nabla \times \boldsymbol{A}
$$

can be expressed as

$$
\begin{aligned}
& B_{r}=-\sum_{n, m} \frac{1}{\rho} n(n+1) f_{n}(r) S_{n}^{m}(\theta, \phi) \\
& B_{\theta}=-\sum_{n, m} \frac{1}{\rho} \frac{\mathrm{d}}{\mathrm{d} r}\left[r f_{n}(r)\right] \frac{\partial S_{n}^{m}(\theta, \phi)}{\partial \theta}
\end{aligned}
$$

and

$$
B_{\phi}=-\sum_{n, m} \frac{1}{\rho} \frac{\mathrm{d}}{\mathrm{d} r}\left[r f_{n}(r)\right] \frac{\partial S_{n}^{m}(\theta, \phi)}{\sin \theta \partial \phi}
$$

In these expressions

$$
f_{n}(r)=C \rho^{n} F_{n}\left(k^{2} r^{2}\right)+D \rho^{-n-1} G_{n}(k r) .
$$

$C$ and $D$ are arbitrary constants and $F_{n}$ and $G_{n}$ are respectively defined by 


$$
F_{n}\left(k^{2} r^{2}\right)=\frac{(2 n+1) !}{2^{n} \cdot n !}(k r)^{-n}\left[e^{k r} \rho_{n}(k r)+(-1)^{n} e^{-k r} \rho_{n}(-k r)\right]
$$

and

$$
G_{n}(k r)=\frac{-2^{n} \cdot(n-1) !}{(2 n-1) !}(k r)^{n+1} e^{-k r} \rho_{n}(-k r) .
$$

$F_{n}$ and $G_{n}$ also obey the following recurrence formulae

$$
\begin{aligned}
& F_{n-1}=F_{n}+\frac{r}{2 n+1} \frac{\mathrm{d} F_{n}}{\mathrm{~d} r} \\
& G_{n+1}=G_{n}-\frac{r}{2 n+1} \frac{\mathrm{d} G_{n}}{\mathrm{~d} r}
\end{aligned}
$$

while $\rho_{n}$ can be expanded in series as below

$$
\rho_{n}(x)=\frac{1}{2 x}-\frac{n(n+1)}{(2 x)^{2}}+\frac{(n-1) n(n+1)(n+2)}{2 !(2 x)^{3}} \cdots .
$$

A2. Evaluation of the field induced by an external current system of known geometry in a sphere of uniform conductivity

At the spherical boundary $r=q a$ or $\rho=q$ (see Fig. A1) between conducting and non-conducting regions, $B_{r}, H_{\theta}$ and $H_{\phi}$ must be continuous. Since the internal field has to remain finite at $r=0$ (center of the conducting sphere) $f_{n}(r)$ (given by Eq. A.7) reduces to

$$
f_{n}(r)=C \rho_{n} F_{n}\left(k^{2} r^{2}\right) .
$$

Assuming then that $\mu$, the magnetic permeability, varies so little from one medium to the other that it can be considered as constant $\left(\mu=\mu_{0}\right)$, the continuity of $B_{r}, H_{\theta}$ and $H_{\phi}$ at $r=q a$ leads to

and

$$
\mu_{0}\left[n E_{n}^{m}-(n+1) q^{-2 n-1} I_{n}^{m}\right]=n(n+1) C F_{n}\left(q^{2} a^{2}\right)
$$

$$
\mu_{0}\left[E_{n}^{m}+I_{n}^{m} q^{-2 n-1}\right]=C\left[-n F_{n}\left(q^{2} a^{2}\right)+(2 n+1) F_{n-1}\left(q^{2} a^{2}\right)\right]
$$

(using Eqs. (A1) to (A6) and because of the orthogonality of the spherical harmonics). Eliminating $C$ between Eqs. (A13) and (A.14), we obtain

$$
I_{n}^{m}=q^{2 n+1} \frac{n}{n+1}\left[1-\frac{F_{n}\left(q^{2} a^{2}\right)}{F_{n-1}\left(q^{2} a^{2}\right)}\right] E_{n}^{m}
$$

i.e. a general relation which allows the determination of the induced field coefficients (at the earth's surface) from the knowledge of the direct field coefficients at the same point.

If the real part of $k r$ is large and positive, $F_{n}$ (given by Eq. (A8)), can be approximated by 


$$
F_{n}\left(k^{2} r^{2}\right)=\frac{1}{(k r)^{n}} \frac{(2 n+1) !}{2^{n} \cdot n !} e^{k r} \rho_{n}(k r) .
$$

This approximation, which is almost always legitimate when the earth is concerned, reduces the relation between induced and direct coefficients (Eq. (A15)) to

$$
I_{n}^{m}=q^{2 n+1} \frac{n}{n+1}\left[1-\frac{(2 n+1)}{k r} \frac{\rho_{n}}{\rho_{n-1}}\right]_{r=q a} E_{n}^{m}
$$

or, if $n$, the degree of the harmonics is quite smaller than Real $(k r)$ to

$$
I_{n}^{m}=q^{2 n+1} \frac{n}{n+1}\left[1-\frac{(2 n+1)}{k r}+\frac{n(2 n+1)}{(k r)^{2}}-\frac{(n-1) n(2 n+1)}{2 !(k r)^{3}}+\cdots\right]_{r=q a} E_{n}^{m}
$$

Zeroth order approximation: the perfect conductor

If either the conductivity of the sphere is high enough or the variation of the source field rapid enough to make

$$
\frac{(2 n+1)}{\operatorname{Real}(k r)} \ll 1 \quad(\text { at } r=q a)
$$

the relation between induced and direct coefficients (Eq. A17) reduces to

$$
I_{n}^{m}=q^{2 n+1} \frac{n}{n+1} E_{n}^{m} .
$$

In this case, the induced field components can be very simply expressed as a function of the direct field components at the same point on the surface of the sphere. For instance $B_{r}^{i}$, defined by Eq. (A.1') becomes

or

$$
\begin{aligned}
B_{r}^{i}(r=q a, \theta, \phi) & =\mu_{0} \sum_{n, m}(n+1) q^{-2-n} I_{n}^{m} S_{n}^{m}(\theta, \phi) \\
& =\mu_{0} \sum_{n, m} n q^{n-1} E_{n}^{m} S_{n}^{m}(\theta, \phi)
\end{aligned}
$$

$$
B_{r}^{i}(r=q a, \theta, \phi)=-B_{r}^{e}(r=q a, \theta, \phi) .
$$

Similarly, after some algebraic manipulations, we have

$$
B_{\theta, \phi}^{i}(r=q a, \theta, \phi)=B_{\theta, \phi}^{e}(r=q a, \theta, \phi)-\frac{1}{a^{2} q^{2}} \int_{0}^{q a} r B_{\theta, \phi}^{e}(r, \theta, \phi) \mathrm{d} r .
$$

Note that in general $q=1$ because the surface of the earth is selected as surface of reference for the spherical harmonics.

First order approximation

If the magnitude of $(2 n+1) /$ Real $(k q a)$ cannot be neglected when compared to 1 but is, nevertheless, large when compared to the magnitude of $\{n(2 n+1)\} /$ Real $(k q a)^{2}$ and following terms, we can determine a first order correction to the induced field components given by Eqs. (A19), (A20) and (A21). 
In this case, the relation between induced and direct coefficients becomes

$$
I_{n}^{m}=q^{2 n+1} \frac{n}{n+1}\left[1-\frac{2 n+1}{k r}\right]_{r=q a} E_{n}^{m}
$$

and by exactly the same technique as previously applied to the zeroth approximation, we obtain, for the first order correctons only

and

$$
B_{r}^{i(1 \mathrm{st})}(r=q a, \theta, \phi)=\frac{2}{k q a}\left[\frac{1}{r^{1 / 2}} \frac{\mathrm{d}}{\mathrm{d} r} r^{3 / 2} B_{r}^{e}(r, \theta, \phi)\right]_{r=q a}
$$

$$
\begin{aligned}
B_{\theta, \phi}^{i(1 \mathrm{st})}(r=q a, \theta, \phi)= & \frac{1}{k q a}\left\{\left[B_{\theta, \phi}^{e}(r, \theta, \phi)-2 \frac{\mathrm{d}}{\mathrm{d} r} r B_{\theta, \phi}^{e}(r, \theta, \phi)\right]_{r=q a}\right. \\
& \left.-\frac{1}{q^{2} a^{2}} \int_{0}^{q a} r B_{\theta, \phi}^{e}(r, \theta, \phi) \mathrm{d} r\right\} .
\end{aligned}
$$

\section{Second order approximation}

If the model selected requires the inclusion of second order terms, the relation between $I_{n}^{m}$ and $E_{n}^{m}$ becomes

$$
I_{n}^{m}=q^{2 n+1} \frac{n}{n+1}\left[1-\frac{(2 n+1)}{k r}+\frac{n(2 n+1)}{(k r)^{2}}\right]_{r=q a} E_{n}^{m} .
$$

In this case, the second order corrections to add to the field components $(0$ th +1 st order terms $)$ are

$$
B_{r}^{i(2 \mathrm{nd})}(r=q a, \theta, \phi)=\frac{1}{(k q a)^{2}}\left[\frac{\mathrm{d}}{\mathrm{d} r} r B_{r}^{e}(r, \theta, \phi)-2 \frac{\mathrm{d}^{2}}{\mathrm{~d} r^{2}} r^{2} B_{r}^{e}(r, \theta, \phi)\right]_{r=q a}
$$

and

$$
\begin{aligned}
B_{\theta, \phi}^{i(2 \text { nd })}(r & =q a, \theta, \phi) \\
= & \frac{1}{(k q a)^{2}}\left[B_{\theta, \phi}^{e}(r, \theta, \phi)+2 r \frac{\mathrm{d}^{2}}{\mathrm{~d} r^{2}} r B_{\theta, \phi}^{e}(r, \theta, \phi)+\frac{\mathrm{d}}{\mathrm{d} r} r B_{\theta, \phi}^{e}(r, \theta, \phi)\right. \\
& \left.-\frac{1}{q^{2} a^{2}} \int_{0}^{q a} r B_{\theta, \phi}^{e}(r, \theta, \phi) \mathrm{d} r\right]_{r=q a} .
\end{aligned}
$$

Obviously, further order terms can be determined by pursuing the expansion of $\rho_{n} / \rho_{n-1}$ (in Eq. (A16)). This will not be done here. The validity of the approximations presented in this appendix is tested in section 4 (text).

Appendix 2. Evaluation of the Field Induced by an External Current System of Known Geometry in a Two-Layered Sphere

Once again, the fundamentals of the method used in this appendix can be found in Chapman and Bartels (1940, p. 732-741). Since they have been summarized in section A1 (Appendix 1), they will not be repeated here. 
The solution is primarily intended for current systems varying slowly with time and therefore inducing currents which can flow quite deeply below the earth's surface. In that case, it may be necessary to simulate the earth by a two-layered sphere, taking into account the jump in conductivity present roughly $500 \mathrm{~km}$ below the surface. To satisfy that purpose, the earth model presented here consists thus of a highly conducting core surrounded by a shell of lower conductivity. (Note, however, that the same model could be used to simulate the high frequency response of a conducting strip close to the earh's surface).

Let $q_{1} a$ be the radius of the Earth and $q_{2} a$ be the radius of the spherical boundary between conducting the highly-conducting regions (see Fig. A2). In region 1 (the surrounding shell), the field components have both increasing and decreasing terms in $r$. We can no longer neglect $G_{n}(k r)$ in the expression of $f_{n}(k r)$ (see Eq. (A7)). However, we can still approximate $F_{n}$ by

$$
F_{n}\left(k^{2} r^{2}\right)=\frac{(2 n+1) !}{2^{n} \cdot n !}(k r)^{-n} e^{k r} \rho_{n}(k r) \text {. }
$$

In region 2 (the inner sphere), we must write, as in section A2 (Appendix 1), that

$$
f_{n}(r)=\frac{C \rho_{n}}{(k r)^{n}} \frac{(2 n+1) !}{2^{n} \cdot n !} e^{k r} \rho_{n}(k r) .
$$

We will again assume that $\mu$ is constant through the discontinuities $\left(\mu=\mu_{0}\right.$ everywhere). Under such assumptions, the boundary conditions on $B_{r}, H_{\theta}$ and $H_{\phi}$ become at $r=q_{1} a$

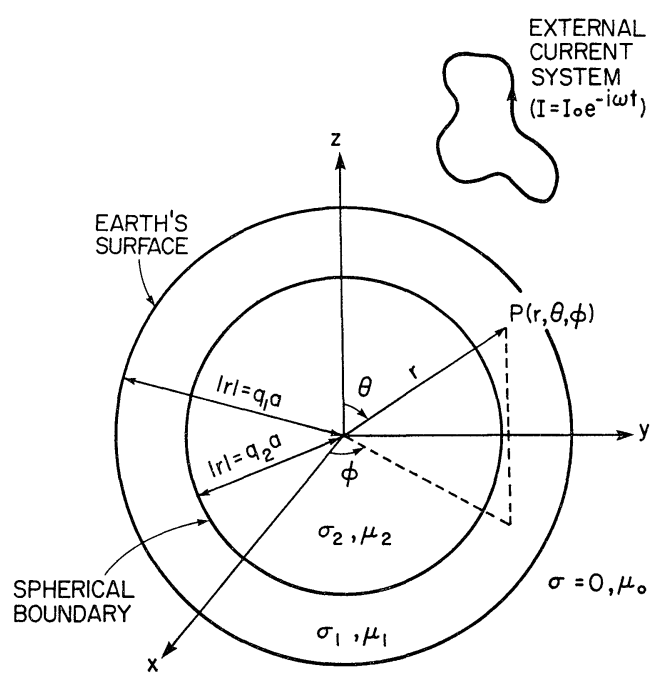

Fig. A2. Cross-section of a two-layered earth with indication of the observation point. 
and

$$
\mu_{0}\left[n q_{1}^{n-1} E_{n}^{m}-(n+1) q_{1}^{-n-2} I_{n}^{m}\right]=\frac{1}{q_{1}} n(n+1) f_{n}^{1}(k r)
$$

$$
\mu_{0}\left[E_{n}^{m} q_{1}^{n-1}+I_{n}^{m} q_{\mathrm{l}}^{-n-2}\right]=\frac{1}{q_{1}}\left[\frac{\mathrm{d}}{\mathrm{d} r}\left(r f_{n}^{1}(k r)\right)\right]
$$

at $r=q_{2} a$

and

$$
f_{n}^{1}(k r)=f_{n}^{2}(k r)
$$

$$
\frac{\mathrm{d}}{\mathrm{d} r}\left(r f_{n}^{1}(k r)\right)=\frac{\mathrm{d}}{\mathrm{d} r}\left(r f_{n}^{2}(k r)\right)
$$

(where the indices 1 or 2 refer to the region in which the function must be evaluated).

These boundary conditions lead (after some algebra) to

where

$$
I_{n}^{m}=q_{1}^{2 n+1} \frac{n}{n+1}\left[\frac{F_{n-1}^{1}-F_{n}^{1}+A G_{n+1}^{1}}{F_{n-1}^{1}+A\left(G_{n+1}^{1}-G_{n}^{1}\right)}\right]_{r=q_{1} a} E_{n}^{m}
$$

$$
A=\left(\frac{q_{2}}{q_{1}}\right)^{2 n+1}\left[\frac{F_{n}^{1} F_{n-1}^{2}-F_{n}^{2} F_{n-1}^{1}}{G_{n}^{1}\left[F_{n-1}^{2}-F_{n}^{2}\right]+F_{n}^{2} G_{n+1}^{1}}\right]_{r=q_{2} a} .
$$

Note that if $k_{2}=k_{1}$ (i.e. $\sigma_{2}=\sigma_{1}$ ), $F^{1}=F^{2}, A=0$ and Eq. (A34) reduces to Eq. (A15), i.e. to the general relation between induced and direct coefficients at the surface of a uniform earth.

If $\operatorname{Real}\left(k_{2} r\right) \gg \operatorname{Real}\left(k_{1} r\right)>n$, we can certainly assume that

$$
\rho_{n}\left(k_{2} r\right) \simeq \rho_{n-1}\left(k_{2} r\right) \simeq \frac{1}{2 k_{2} r} \quad\left(\text { at } r=q_{2} a\right)
$$

and the evaluation of $A$ is much simplified. In this case, we have

where

$$
A=A_{0}\left[\frac{\rho_{n}\left(k_{1} r\right)-\frac{k_{1}}{k_{2}} \rho_{n-1}\left(k_{1} r\right)}{\rho_{n}\left(-k_{1} r\right)\left[\frac{2 n+1}{k_{2} r}-1\right]-\frac{k_{1}}{k_{2}} \rho_{n+1}\left(-k_{1} r\right)}\right]_{r=q_{2} a}
$$

$$
A_{0}=-\left.\frac{(2 n+1) !(2 n-1) !}{2^{n} \cdot 2^{n-1} \cdot n !(n-1) !}\left(\frac{q_{2}}{q_{1}}\right)^{2 n+1} \frac{e^{2 k_{1} r}}{2\left(k_{1} r\right)^{2 n+1}}\right|_{r=q_{2} a} .
$$

Equation (A36) can be further simplified since it is clear that if Real $\left(k_{2} r\right)$ is quite large (the central core is quite conducting),

$$
\frac{2 n+1}{\operatorname{Real}\left(k_{2} r\right)} \ll 1 \text {. }
$$

But since we assume that though Real $\left(k_{2} r\right)$ is much larger than Real $\left(k_{1} r\right)$, 
Real $\left(k_{1} r\right)$ itself is still large compared to $n$ (the surrounding shell is relatively conducting), the series developments of $\rho_{n}\left(k_{1} r\right), \rho_{n-1}\left(k_{1} r\right)$ and $\rho_{n+1}\left(k_{1} r\right)$ can be approximated by their first terms.

Then, $A$ becomes

$$
A=\left.A_{0} \frac{\rho_{n}\left(k_{1} r\right)}{\rho_{n}\left(-k_{1} r\right)}\right|_{r=q_{2} a}
$$

Using that expression to evaluate the induced field coefficient through Eq. (A34), we obtain

$$
I_{n}^{m}=q_{1}^{2 n+1} \frac{n}{n+1}\left[1-\frac{2 n+1}{k_{1} q_{1} a}\left[\frac{e^{2 k_{1} a\left(q_{1}-q_{2}\right)}-1}{e^{2 k_{1} a\left(q_{1}-q_{2}\right)}+1}\right]\right] E_{n}^{m} .
$$

Obviously, if $q_{1}=q_{2}$ i.e. if there is no finitely conducting shell surrounding the central highly conducting core

$$
I_{n}^{m}=q_{1}^{2 n+1} \frac{n}{n+1} E_{n}^{m} .
$$

Thus we see that this more general solution reduces to Eq. (A18), the relation between induced and direct coefficients at the surface of a highly conducting uniform earth.

On the other hand, if $q_{1}$ is much larger than $q_{2}$, the central core is very small and the electrical behaviour of the earth is almost solely governed by the properties of its external shell.

Then

$$
\frac{e^{2 k_{1} \alpha\left(q_{1}-q_{2}\right)}-1}{e^{2 k_{1} \alpha\left(q_{1}-q_{2}\right)}+1} \simeq 1
$$

and Eq. (A.38) reduces to

$$
I_{n}^{m} \simeq q_{1}^{2 n+1} \frac{n}{n+1}\left[1-\frac{2 n+1}{k_{1} r}\right]_{r=q_{1} a} E_{n}^{m}
$$

which is exactly the relation between $I_{n}^{m}$ and $E_{n}^{m}$ (up to the first order terms) observed at the surface of a relatively conducting $\left(\sigma=\sigma_{1}\right)$ uniform earth.

Clearly, Eq. (A.38) allows determinations of the induced field components as easily as in Appendix 1. Indeed, the zeroth order terms are identical to those given by Eqs. (A.19) to (A.21) while the first order corrections are simply.

$$
\begin{aligned}
& B^{i(1 \mathrm{st})}(\text { two-layered model }) \\
= & B^{i(1 \mathrm{st})}(\text { uniform sphere }) \times \frac{e^{2 k_{1} a\left(q_{1}-q_{2}\right)}-1}{e^{2 k_{1} a\left(q_{1}-q_{2}\right)}+1} .
\end{aligned}
$$

The range of validity of this simple result is discussed in section 4 of the 
text. Though approximations of higher order could theoretically be calculated, they would involve much more complicated expressions than Eq. (A.38) since the terms neglected under the assumption that Real $\left(k_{1} r\right)>n$ would have to be retained until the final result.

One of the authors (M.M.) acknowledges financial support from the National Research Council of Canada while pursuing this work.

\section{REFERENCES}

Andersen, H.R. and R.R. Vondrak, Observations of Birkeland currents at auroral latitudes, Rev. Geophys. Space Phys., 13, 243-262, 1975.

Armstrong, J.C. and A.J. Zmuda, Triaxial magnetic measurements of field-aligned currents at 800 kilometers in the auroral region: Initial results, J. Geophys. Res., 78, 6802-6807, 1973.

Ashour, A.A., The evaluation of the field of the currents induced in the Earth by an external field whose distribution is known numerically, Radio Sci., 6, 171-173, 1971.

BANKS, R.J., The overall conductivity distribution of the earch, J. Geomag. Geoelecr., 24, 337351, 1972.

Bonnevier, B., R. Boström, and G. Rostoker, A three-dimensional model current system for polar magnetic substorms, J. Geophys. Res., 75, 107-122, 1970.

Chapman, S. and J. Bartels, Geomagnetism, p. 732-741, Clarendon Press, Oxford, 1940.

Chappell, C.R., Conference on magnetospheric-ionospheric coupling, E.O.S. Trans., 55, 776, 1974.

Cloutier, P.A., Ionospheric effects of Birkeland currents, Rev. Geophys. Space Phys., 9, 987996, 1971.

Czechowsky, P., Calculation of an equivalent current system in the polar E-region, Radio Sci., 6, 247-253, 1971.

Horning, B.L., R.L. MCPherron, and D.D. Jackson, Application of linear inverse theory to a line current model of substorm current systems, J. Geophys. Res., 79, 5202-5210, 1974.

Kisabeth, J.L., The dynamical development of the polar electrojets, Ph.D. Thesis, Univ. of Alberta, Edmonton, Alta., 1972.

Kisabeth, J.L. and M. Mareschal, The theory of the generalized least-squares parameter estimation applied to current systems associated with polar magnetic substorms, E.O.S. Trans., 55, 991, 1974.

KisAbeth, J.L. and G. Rostoker, Development of the polar electrojet during polar magnetic substorms, J. Geophys. Res., 76, 6815-6828, 1971.

Kisabeth, J.L. and G. Rostoker, Current flow in auroral loops and surges inferred from ground-based magnetic observations, J. Geophys. Res., 78, 5573-5584, 1973.

Kisabeth, J.L. and G. Rostoker, The expansive phase of magnetospheric substorms. 1. Devolopment of the auroral electrojets and auroral arc configuration during a substorm, $J$. Geophys. Res., 79, 972-984, 1974.

KisABETH, J.L. and G. RostoKer, Modelling of three-dimensional current systems associated with magnetospheric substorms, Geophys. J.R. Astr. Soc., 49, 655-684, 1977.

LAHIRI, B.N. and A.T. Price, Electromagnetic induction in non-uniform conductors and the determination of the conductivity of the Earth from terrestrial magnetic variations, Philos. Trans. R. Soc. London, Ser. A, 237, 509-540, 1939.

Mareschal, M., On the problem of simulating the earth's induction effects in modeling polar magnetic substorms, Rev. Geophys. Space Phys., 14, 403-409, 1976. 
Nopper, R.W. and J.F. Hermance, Phase relations between polar magnetic substorm fields at the surface of a finitely conducting Earth, J. Geophys. Res., 79, 4799-4804, 1974.

OldenburG, D.W., Ionospheric current structure as determined from ground-based magnetometer data, Geophys. J.R. Astr. Soc., 46, 41-60, 1976.

Potemra, T.A. and T. Inima, Characteristics of large scale field aligned currents: Possible source mechanisms, AGU International Symposium on Solar-Terrestrial Physics, Boulder, Colorado, 1976.

Rostoker, G. and J.L. Kisabeth, The response of the polar electrojets in the evening sector to polar magnetic substorms, J. Geophys. Res., 78, 5559-5571, 1973. 